\title{
Characterization of primary normal and malignant breast cancer cell and their response to chemotherapy and immunostimulatory agents
}

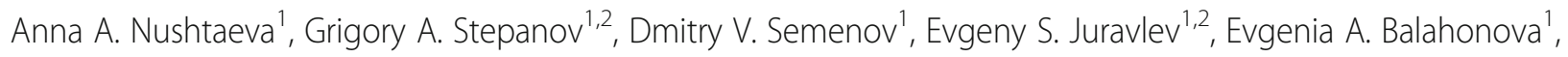
Alexey V. Gerasimov ${ }^{3}$, Sergey V. Sidorov ${ }^{4}$, Eugeniy I. Savelyev ${ }^{5}$, Elena V. Kuligina', Vladimir A. Richter ${ }^{1}$ and Olga A. Koval ${ }^{1,2^{*}}$ (D)

\begin{abstract}
Background: The phenomenon of chemotherapy-resistant cancers has necessitated the development of new therapeutics as well as the identification of specific prognostic markers to predict the response to novel drugs. Primary cancer cells provide a model to study the multiplicity of tumourigenic transformation, to investigate alterations of the cellular response to various molecular stimuli, and to test therapeutics for cancer treatment.

Methods: Here, we developed primary cultures of human breast tissue - normal cells (BN1), cancer cells (BC5), and cells from a chemotherapy-treated tumour (BrCCh1) to compare their response to conventional chemotherapeutics and to innate immunity stimulators with that of the immortalized breast cells MCF7, MDA-MB-231, and MCF10A. Expression of the progesterone receptor (PGR), oestrogen receptor (ER) a and $\beta$, human epidermal growth factor receptor (HER) 2 and 3 and aromatase CYP19, as well as expression of interferon-induced protein with tetratricopeptide repeats 3 (IFIT3) mRNA in human breast cells were characterized.

Results: We revealed that BC5 carcinoma cells were PGR ${ }^{\text {low }} / \mathrm{ERb}^{\text {high }} /$ ERa ${ }^{-} / C y p 19^{+}$, the BrCCh1 cells that originated from the recurrent tumour were $\mathrm{PGR}^{-} / \mathrm{ERb}^{+} / \mathrm{ERa}^{-} / \mathrm{Cyp} 19^{+}$, and normal BN cells were $\mathrm{PGR}{ }^{-} / \mathrm{ERb}^{+} / \mathrm{ERa}^{-} / \mathrm{Cyp} 19^{\text {high }}$. The treatment of primary culture cells with antitumour therapeutics revealed that BrCCh1 cells were doxorubicine-resistant and sensitive to cisplatin. BC5 cells exhibited low sensitivity to tamoxifen and cisplatin. The innate immunity activators interferon-a and an artificial small nucleolar RNA analogue increased expression of IFIT3 at different levels in primary cells and in the immortalized breast cells MCF7, MDA-MB-231, and MCF10A. The relative level of activation of IFIT3 expression was inversely correlated with the baseline level of IFIT3 mRNA expression in breast cell lines.
\end{abstract}

Conclusion: Our data demonstrated that primary cancer cells are a useful model for the development of novel cancer treatments. Our findings suggest that expression of IFIT3 mRNA can be used as a prognostic marker of breast cancer cell sensitivity to immunostimulating therapeutics.

Keywords: Breast cancer, Primary culture, Hormone receptor, Prognostic marker, Cancer stem cells, CD44, CD24, IFIT3, Interferon-a, snoRNA

\footnotetext{
* Correspondence: o_koval@ngs.ru

${ }^{1}$ Institute of Chemical Biology and Fundamental Medicine, Siberian Branch,

Russian Academy of Sciences, Lavrentiev Avenue, 8, 630090 Novosibirsk,

Russia

${ }^{2}$ Novosibirsk State University, Pirogova str., 1, 630090 Novosibirsk, Russia

Full list of author information is available at the end of the article
}

(c) The Author(s). 2018 Open Access This article is distributed under the terms of the Creative Commons Attribution 4.0 International License (http://creativecommons.org/licenses/by/4.0/), which permits unrestricted use, distribution, and

reproduction in any medium, provided you give appropriate credit to the original author(s) and the source, provide a link to the Creative Commons license, and indicate if changes were made. The Creative Commons Public Domain Dedication waiver (http://creativecommons.org/publicdomain/zero/1.0/) applies to the data made available in this article, unless otherwise stated. 


\section{Background}

Breast cancer was the leader among new cancer cases in the United States in 2016 and the second highest cause of death among women [1]. Cancer development is a multi-step process where various oncogenic mutations give rise to cancer cells with different genetic defects, which can differ even within an individual tumour. This diversity is a significant obstacle in cancer treatment, so cell lines established from human tumour samples can be a beneficial tool for screening relevant drugs. For prognosis of cancer sensitivity to certain therapeutics, direct cytotoxicity assays in cancer cells can be combined with data regarding the expression of genes essential for antitumour action [2]. The significant characteristics of individual breast cancers are the expression of oestrogen, progesterone, and human epidermal growth factor receptor 2 (HER2/CD340), and the proposed type of treatment is based in part on these characteristics [3]. Among them, oestrogen receptor (ER) status is more critical for predicting the response to hormonal therapy, whereas progesterone receptor (PGR) status has remained controversial $[4,5]$. Double-positive $\mathrm{ER}^{+} / \mathrm{PGR}^{+}$breast cancer has shown better outcomes than single-positive tumours. HER2 expression allows for the choice of anti-HER2 targeted therapy, whereas the triple negative breast cancer (TNBC) subtype is usually more aggressive, with the worst prognosis, as targeted or hormonal therapy is not available and patients are treated with standard chemotherapy or radiotherapy [6].

A novel approach to cancer therapy arises from the existence of cancer stem cells (CSCs), which display a drug resistant phenotype. It has been reported that recurrent breast tumours are driven by a subpopulation of tumour-initiating CSCs. A subpopulation of cancer cells with a $\mathrm{CD} 44^{+} / \mathrm{CD} 24^{-/ \text {low }}$ phenotype has stem/progenitor cell features that are associated with a poor outcome in patients $[7,8]$. In vitro screening of drug sensitivity can help to reduce the chemotherapeutic dose and decrease the toxic effects of low-effective chemotherapeutics, including TNBC subtypes and CSC-reach cancers. This approach can decrease the immunosuppressive effects of chemotherapy and as a consequence, the immune system would recognize transformed cells in order to inhibit the growth of neoplastic tissue [9]. In such way immunosurveillance possible to make contribution against cancer.

Over the past few years, immunotherapy has become a part of a complex approach for the treatment of malignant diseases. In addition to new T-cell adoptive transfer and monoclonal antibodies, interferons (IFNs) and cytokines can produce therapeutic responses $[10,11]$. IFN $\alpha$ is used to treat hairy-cell leukaemia, renal carcinoma, myeloma, and melanoma, whereas IFNy has been approved for the treatment of ovarian, renal, and endometrial cancers [12]. Because of the broad spectrum of individual side effects and the toxicity that accompany IFN therapy, new molecules with immunomodulatory properties that can trigger innate immunity and initiate apoptosis of cancer cells are being studied.

RNAs have been shown to modulate various cellular responses as well as induce apoptosis of cancer cells in vitro $[13,14]$. Recently, it was demonstrated that the level of expression of hepatic interferon-induced protein with tetratricopeptide repeats 3 (IFIT3) mRNA predicts the IFN $\alpha$ therapeutic response in patients with hepatocellular carcinoma [15]. Here, we used IFN $\alpha$ and an artificial analogue of U25 small nucleolar RNA (snoRNA) to modulate expression of innate immunity genes in breast cells and determined that activation of IFIT3 expression is inversely correlated with its mRNA baseline level in primary breast cells and in immortalized breast cell lines.

\section{Methods \\ Chemicals and antibodies}

Cisplatin, doxorubicin, anastrozole, exemestane, and everolimus (afinitor) were purchased from Sigma-Aldrich (St. Louis, MO, USA). Phycoerythrin (PE)-conjugated mouse anti-human CD44 monoclonal (\#MHCD4404) and fluorescein isothiocyanate (FITC)-conjugated mouse anti-human CD24 monoclonal (\#MHCD4201) antibodies were purchased from Molecular Probes (Invitrogen, Carlsbad, CA, USA). FITC-conjugated mouse anti-human HER2 monoclonal and allophycocyanin (APC)-conjugated mouse anti human HER3 monoclonal (\#2223535) antibodies were purchased from Sony Biotechnology Inc. (San Jose, CA, USA). APC- and FITC-conjugated IgG controls were from BD Biosciences.

\section{Cell cultures}

MCF7, MDA-MB-231, and MCF10A cells were obtained from the Russian cell culture collection (Russian Branch of the ETCS, St. Petersburg, Russia). MDA-MB-231 cells were grown in Leibovitz media (L15, Sigma-Aldrich) supplemented with $10 \%$ fetal bovine serum (FBS; Gibco BRL Co., Gaithersburg, MD, USA), 2 mM L-glutamine, $250 \mathrm{mg} / \mathrm{mL}$ amphotericin B, and $100 \mathrm{U} / \mathrm{mL}$ penicillin/ streptomycin. MCF7 cells were cultivated in Iscove's modified Dulbecco's media (IMDM; Sigma-Aldrich) with 10\% FBS (Gibco BRL Co., Gaithersburg, MD, USA), $2 \mathrm{mM}$ L-glutamine (Sigma-Aldrich), $250 \mathrm{mg} / \mathrm{mL}$ amphotericin $\mathrm{B}$, and $100 \mathrm{U} / \mathrm{mL}$ penicillin/streptomycin (Gibco BRL Co., Gaithersburg, MD, USA). MCF10A were cultured in HuMEC Basal Serum-free medium (Gibco BRL Co., Gaithersburg, MD, USA) supplemented with HuMEC Supplement Kit (Gibco BRL Co., Gaithersburg, MD, USA). 


\section{Human tissue specimens}

Normal human breast tissue was obtained during size-reduction plastic surgery from healthy women at the Centre of New Medical Technologies (Novosibirsk, Russian Federation). Cancer tissue samples were obtained with informed consent from patients at the Novosibirsk Region Oncologic Dispensary (Novosibirsk, Russian Federation). The final diagnosis of cancer was confirmed by haematoxylin-eosin staining of paraffin blocks after the operation. One of the patients received six courses of chemotherapy with doxorubicin/cyclophosphamide before surgery. All patients gave written informed consent. The study protocol was approved by the Institute of Molecular Biology and Biophysics SB RAS Ethics Committee (Report\#1 from March, 14 2017) in accordance with the Declaration of Helsinki of 1975. The fresh tumour and normal tissue specimens were immediately transferred into ice-cold DMEM medium (Gibco BRL Co., Invitrogen) supplemented with $100 \mathrm{U} /$ $\mathrm{mL}$ penicillin, $100 \mu \mathrm{g} / \mathrm{mL}$ streptomycin, and $250 \mathrm{mg} / \mathrm{mL}$ amphotericin B.

\section{Primary cell culture preparation}

Tissue specimens were mechanically dissociated using a scalpel and transferred to a solution of $20 \mathrm{mg} / \mathrm{mL}$ collagenase I (Gibco BRL Co., Invitrogen) in DMEM medium and incubated at $37{ }^{\circ} \mathrm{C}$ for $15 \mathrm{~h}$ on a shaking incubator (Grant Bio, Keison Products, UK). Specimens dissociated into single cells were washed with $10 \times$ excess of phosphate-buffered saline (PBS) and separated cells were collected by centrifugation at $300 \times g$. Cells were plated in IMDM with $10 \% \mathrm{FBS}$ and, after cell adhesion, $10 \mu \mathrm{M}$ Rho-associated protein kinase (ROCK) inhibitor was added to the culture medium for $1 \mathrm{~h}$ and the medium in the plates was replaced with fresh complete IMDM medium. At the next passages, cells were cultured in complete IMDM medium supplemented with epithelial cell growth supplement (\#6622, Cell Biologics, Chicago, IL, USA), Mito + Serum Extender (BD Biosciences - Discovery Labware, San Jose, CA, USA), 2 mM L-glutamine, $100 \mathrm{U} / \mathrm{mL}$ penicillin, $100 \mu \mathrm{g} / \mathrm{mL}$ streptomycin, and $250 \mathrm{mg} / \mathrm{mL}$ amphotericin $\mathrm{B}$ and were cultivated in 6-well plates at $37{ }^{\circ} \mathrm{C}$ in a humidified atmosphere containing 5\% $\mathrm{CO}_{2}$. When 70-80\% confluence was reached, cells were harvested using $0.05 \%$ trypsin/ethylenediaminetetraacetic acid (Sigma-Aldrich) and sub-cultured for further experiments.

\section{Cytotoxicity assay}

Cell proliferation and survival were analysed using the iCELLigence RTCA (Real Time Cell Analyser) system by measuring cell-to-electrode responses of the cells seeded in 8-well E-plates with the integrated microelectronic sensor arrays (ACEA Biosciences Inc., San Diego, CA,
USA). The functional unit of a cellular impedance assay is a set of gold microelectrodes fused to the bottom surface of plate well. The assay was performed as reported previously [16]. Briefly, cells were seeded in 8-well electronic plates at a density of about 1500 cells per well in a total volume of $200 \mu \mathrm{L}$ of IMDM and were monitored in real-time mode with the iCELLigence RTCA system. After the initial $24 \mathrm{~h}$ of growth, the culture medium was replaced with fresh medium with dissolved drugs and monitored real-time. Cell index was calculated for each E-plate well by RTCA Software 1.2 (Roche Diagnostics, Meylan, France). Cell index is a parameter reflecting the impedance of electron flow caused by adherent cells. When cells reach the confluence, the $\mathrm{CI}$ value reaches the plateaus.

\section{Flow cytometry}

Cells growing in 6-well plates were collected, fixed in $10 \%$ neutral buffered formalin, and incubated with labelled mouse anti-human antibodies (CD24 and CD44 or HER2/CD340 and HER3) for $30 \mathrm{~min}$ on ice in PBS supplemented with $10 \%$ normal goat serum. All analyses were performed using a FACSCantoII flow cytometer (BD Biosciences, Franklin Lakes, NJ, USA), and the data were analysed by FACSDiva Software (BD Biosciences). Cells were initially gated based on forward versus side scatter to exclude small debris, and ten thousand events from this population were collected. Control cells were treated with appropriate isotype FITC and APC-conjugated IgG (BD Biosciences).

RNA transfection, INFa exposure, and total RNA isolation Cells were seeded at $5 \times 10^{4}$ cells/well in a 24-well plate and incubated for $24 \mathrm{~h}$ in a humidified incubator at $37{ }^{\circ} \mathrm{C}$ with $5 \% \mathrm{CO}_{2}$. Analogues of snoRNAs were preincubated with Lipofectamine 3000 (Invitrogen) according to the manufacturer's protocol and added to the culture medium at a final concentration of $50 \mathrm{nM}$. INF $\alpha$ was added to the culture medium at a final concentration of $40 \mathrm{IU} / \mathrm{mL}$. After incubation for $21 \mathrm{~h}$, total RNA was isolated by phenol-chloroform extraction using the Lira reagent (Biolabmix Ltd., Novosibirsk, Russia) according to the manufacturer's protocol. The quality of total RNA was assessed by agarose gel electrophoresis or capillary electrophoresis with an Agilent 2100 Bioanalyzer, using $28 \mathrm{~S} / 18 \mathrm{~S}>2$ or RIN $>8.0$ criterion.

\section{Quantitative reverse transcription polymerase chain reaction ( $R T-P C R)$}

RT-PCR was performed in the one-tube reaction mixture BioMaster RT-PCR SYBR Blue (Biolabmix Ltd., Novosibirsk, Russia, www.biolabmix.ru) with gene-specific primers:

ER $\alpha$ : 5'-ATGATGAAAGGTGGGATACGA-3' and 5'-CT GTTCTTCTTAGAGCGTTTGATC-3'; ER $\beta$ : 5' ${ }^{\prime}$-TTGG ATGGAGGTGTTAATGATG $-3^{\prime}$ and 5'-GAAGTAGTT 
GCCAGGAGCATGT-3'; PGR: 5'TCATTCTATTCATT ATGCCTTACCA-3' and 5'-GACTTCGTAGCCCTTCCA AAG-3';Cyp19: 5'-TGCGAGTCTGGATCTCTGGA-3' and 5'-GGGCCTGACAGAGCTTTCATA-3'; hypoxanthineguanine phosphoribosyltransferase (HPRT): 5'-CATC AAAGCACTGAATAGAAAT-3' and 5'-TATCTTCCAC AATCAAGACATT-3'; glyceraldehyde 6-phosphate dehydrogenase (GAPDH): 5'-GAAGATGGTGATGGGATT TC-3' and 5'-GAAGGTGAAGGTCGGAGT-3'; U6: 5'-G TGCTCGCTTCGGCAGCAC-3' and 5'-GGGCCATGC TAATCTTCTC-3'; and IFIT3: 5'-GGCAGACAGGAAGA CTTCTG-3' and 5'-TTTCTGCTTGGTCAGCATGT-3'.

To compare PCR product yields, we performed real-time RT-PCR on a Bio-Rad iQ5 Cycler (Hercules, CA, USA) and Light Cycler 96 (Roche, Roche Diagnostics International, Switzerland) and used common models and algorithms for analysis of real-time PCR data realized in corresponding equipment software [17-19]. The quality of reference genes was assessed using geNorm (Qbase + ). Mean values ( \pm standard deviation) from three independent experiments are shown.

\section{Statistical analysis}

Significance was determined using a two-tailed, Student's $t$-test using OriginPro 2015 software. All error bars represent standard error of the mean.

\section{Results}

Cell cultures were established from the malignant breast tissue obtained after surgery by enzymatic disaggregation as described in the Methods. Carcinoma cells were prepared from the primary tumour (BC5) and the recurrent tumour after six courses of doxorubicin/cyclophosphamide chemotherapy (BrCCh1) while normal cells (BN1) were prepared from non-transformed breast tissue of healthy women. Morphological characteristics of cells grown for 1 week are presented in Fig. 1.

At this phase of culture, we observed a morphological heterogeneity of cells, such as long, flattened mesenchymal-like cells and epithelioid cells with the presence of the occasional multinucleated cell.
Primary cells were analysed for expression of PGR, ER, HER2, and HER3. To assess whether the established cell cultures express ERs, PGRs, and Cyp19, we analysed their specific mRNA compared with mRNA levels in the ER-positive human breast adenocarcinoma cell line MCF-7. mRNA signals from primary cells were defined as low/high if they were three times or more lower/higher than in MCF-7 cells, respectively. We revealed that $\mathrm{BC} 5$ carcinoma cells were $\mathrm{PGR}^{\text {low }} / \mathrm{ER} \beta^{\text {high }} / \mathrm{ER}^{-} / \mathrm{Cyp} 19^{+}, \mathrm{BrCCh} 1$ cells that originated from the recurrent tumour were $\mathrm{PGR}^{-} / \mathrm{ER} \beta^{+}$/ $\mathrm{ER \alpha} \alpha^{-} / \mathrm{Cyp} 19^{+}$, and normal BN1 cells were $\mathrm{PGR}^{-} /$ $\mathrm{ER} \beta^{+} / \mathrm{ER \alpha}^{-} /$Cyp $19^{\text {high }}$ (Fig. 2a).

Expression of ER $\beta$ mRNA was 16-times higher in BC5 cells than MCF-7 cells. The expression of aromatase mRNA in BC5 and BrCCh1 cells was similar and match with aromatase mRNA expression in MCF-7 cells while BN1 cells expressed Cyp19A1 mRNA at a high level. $E R \alpha$ mRNA was not detected in all analysed cell cultures that is characterized these cells as ER $\alpha$-negative (Fig. 2a).

Overexpression of the tyrosine kinases HER2 (CD340) and HER3 is associated with decreased overall survival in breast cancer. Thus, these receptors play an important role in breast cancer. The HER2/HER3 heterodimer is a critical oncogenic unit associated with reduced relapse-free and decreased overall survival [20]. Here, HER2 and HER3 surface expression in $\mathrm{BC} 5$ and BrCCh1 cells was analysed by flow cytometry. Positivity for HER2 was defined as more than $10 \%$ cells stained for HER2, according to ASCO guideline, and for HER3 the cutoff value was the same [21]. We found that only BrCCh1 cells contain an essential $\mathrm{HER}^{+}$population $(\approx 20 \%)$ and a HER2 ${ }^{+} /$ $\mathrm{HER}^{+}$double positive population (Fig. $2 \mathrm{~b}$ ). BrCCh1 cells containing about 10\% HER2-positive population were defined low-positive for HER2. Analysis of a CSC-like population in $\mathrm{BC} 5, \mathrm{BrCCh} 1$, and $\mathrm{BN} 1$ cell lines was performed using a CD44/CD24 flow cytometry assay. We determined that approximately $37 \%$ of BrCCh1 cells exhibited a CD $44^{+} / \mathrm{CD} 24^{-}$phenotype, which is associated with a poor outcome in patients [22], but the percentage of $\mathrm{CD} 44^{+} / \mathrm{CD} 24^{-}$cells in

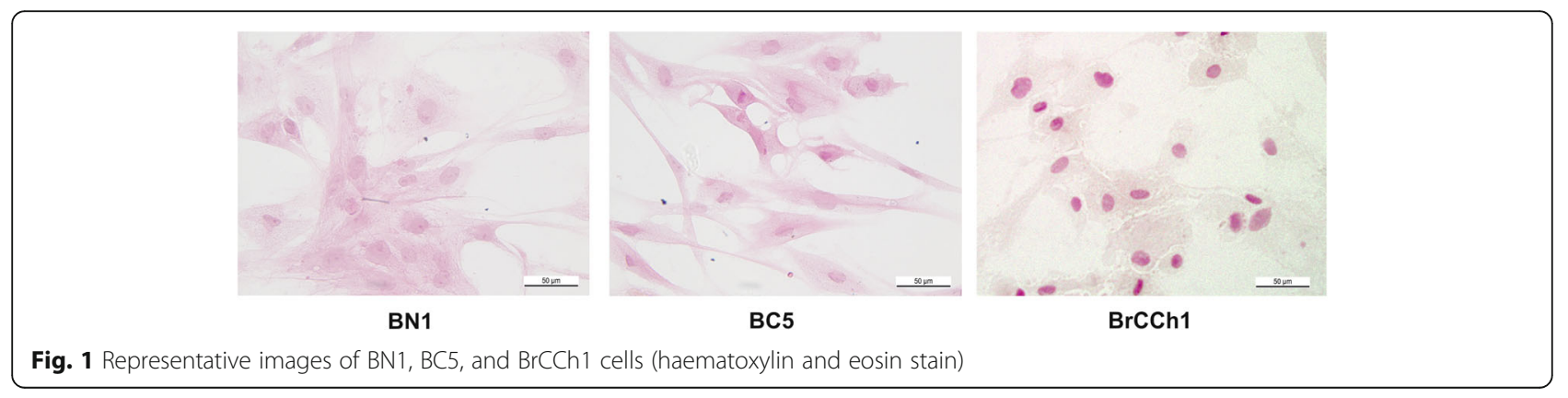



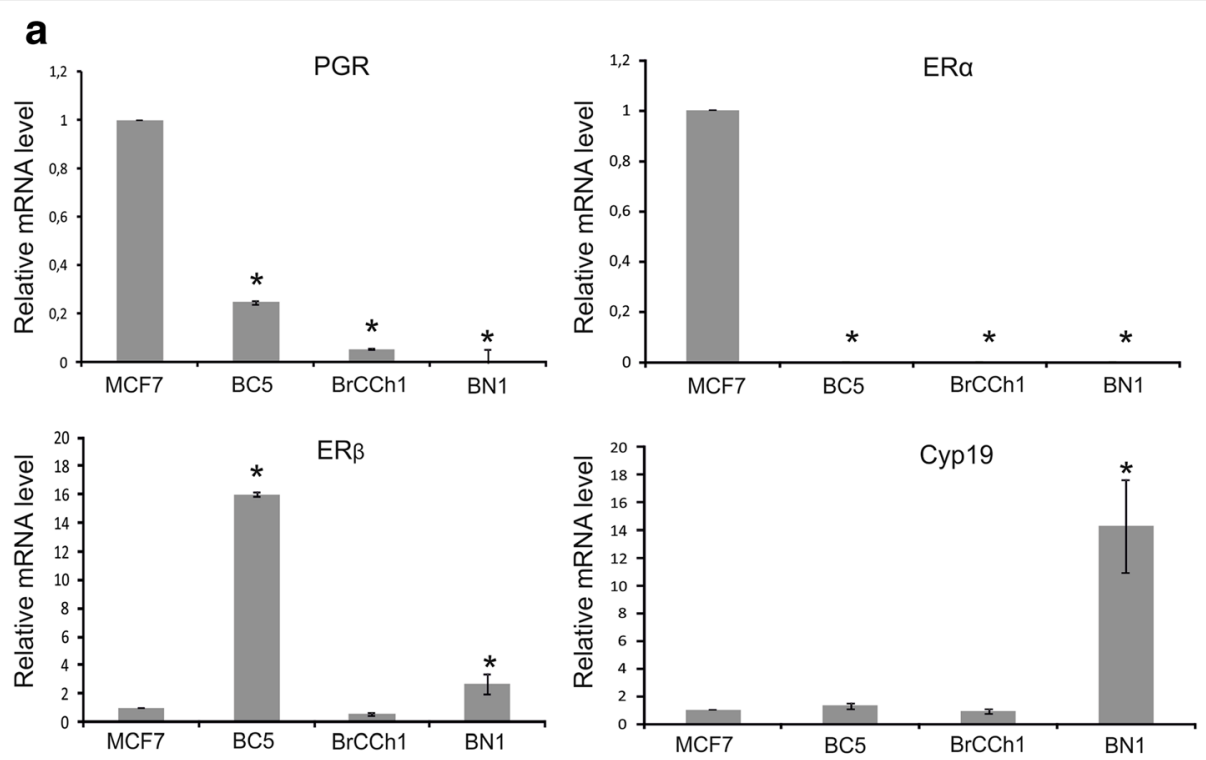

b

BC5
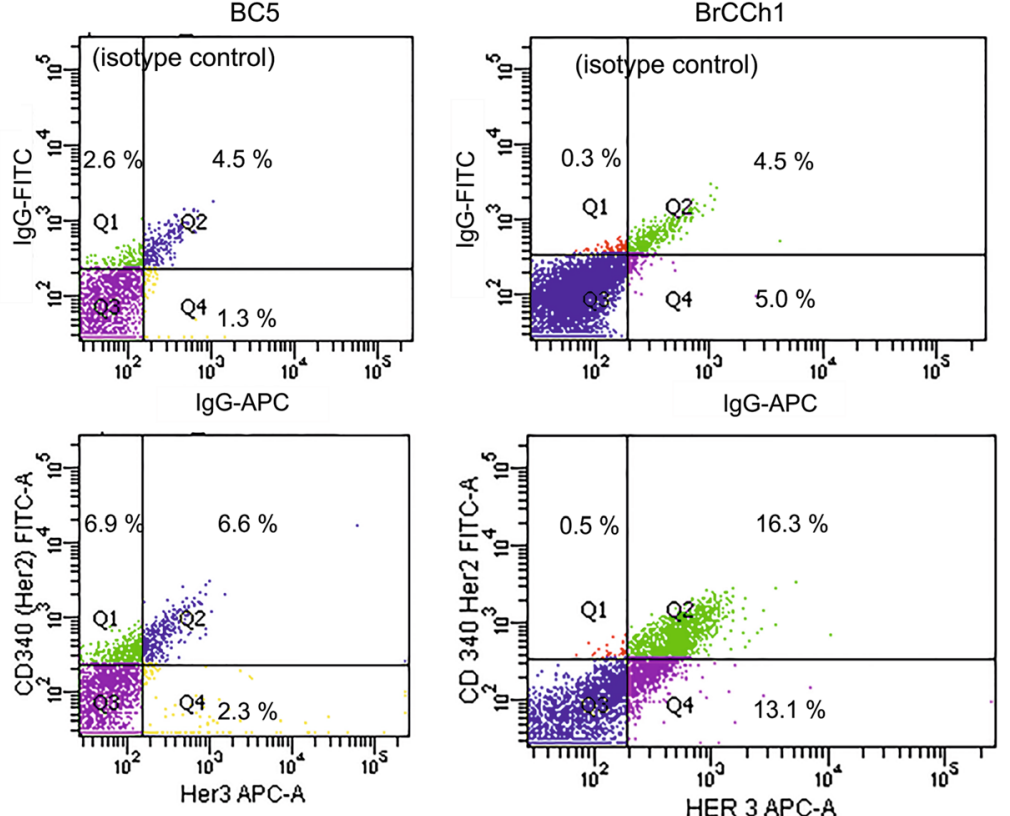

Fig. 2 Expression of biological markers in MCF-7, BC5, BrCCh1, and BN1 cells. a Expression levels of the progesterone receptor (PGR), oestrogen receptor (ER) $a$ and $\beta$, and Cyp19 mRNA in primary cell lines according to real-time polymerase chain reaction (PCR) analysis. The expression of specific mRNAs was normalised to the expression level of glyceraldehyde 6-phosphate dehydrogenase (GAPDH) mRNA. The expression levels of PGR, ERa, ERß, and Cyp19 mRNA in the different cell lines are shown relative to their expression level in MCF-7 cells where it was set equal to 1. Statistical analysis included the results of two independent experiments (mean $\pm \mathrm{SD}$ ). ${ }^{*}$ The difference between the experimental group and the control (MCF-7) group was statistically significant at $p<0.05$. b Representative flow cytometry data of human epidermal growth factor receptor HER2(CD340)/HER3 positive cells in BC5 and BrCCh1 cells. Stained cells were gated according to isotype control samples so that these cells were negative to both HER2/HER3 (left bottom quadrant). Cells from right upper quadrant were accounted as HER2/HER3 double-positive cells

BrCCh1 cells was lower than in MDA-MB-231 cells (Fig. 3).

Next, we estimated the sensitivity of $\mathrm{BC} 5, \mathrm{BrCCh} 1$, and BN1 breast cells to various chemotherapeutic agents: doxorubicin, cisplatin, tamoxifen, anastrozole, and the mTOR-inhibitor afinitor (everolimus), which is used for treatment of advanced hormone receptor-positive, HER2-negative breast cancer [23]. It was determined that nonmalignant BN1 cells were sensitive only to a high dose of doxorubicin (Fig. 4).

Anastrozole is an aromatase inhibitor that actively inhibits oestrogen conversion [24]. Both BN1 normal 


\section{$\mathrm{CD}_{4} 4^{+} / \mathrm{CD} 24$}

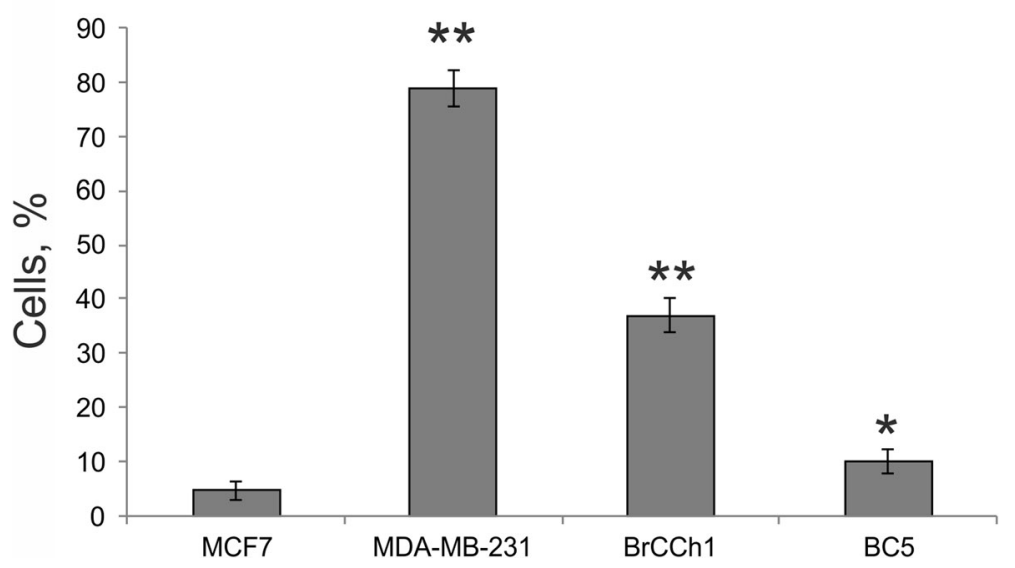

Fig. 3 Relative contribution of the cancer stem cell-like $C D 44^{+} / C D 24^{-}$subpopulations in cultures of breast cancer cells. Bar graph showing the percentage of $\mathrm{CD}_{4} 4^{+} / \mathrm{CD} 24^{-}$cells detected by flow cytometry. Statistical analysis included the results of three independent experiments (mean \pm SD). The difference between the experimental group and the MCF-7 group was statistically significant at $p<0.05\left(^{*}\right)$ and at $p<0.01\left({ }^{* *}\right)$

and BrCCh1 cancer cells were resistant to low concentrations $(50 \mathrm{mkg} / \mathrm{mL})$ of anastrozole. BC5 cells were sensitive to high doses of tamoxifen $(100 \mathrm{mkg} / \mathrm{mL})$ and less sensitive to $5 \mathrm{mkM}$ of cisplatin than BrCCh1 cells (Fig. 4). BrCCh1 cells were sensitive only to $2 \mathrm{mkM}$ of cisplatin, which allowed us to describe these cells as drug-resistant.

Previously, we analysed the influence of artificial small non-coding RNAs on gene expression in cancer cells and determined that transfection of MCF-7 breast cancer cells with artificial analogues of box C/D snoRNAs strongly induced activation of innate immunity genes, including IFIT3 [14]. It is known that the induction of IFN/STAT1-related gene expression, which includes IFIT3, could be an early predictive marker of tumour response to chemotherapy in $\mathrm{ER}^{-}$breast cancers [25].

To characterise the sensitivity of BC5, BrCCh1, and BN1 breast cells to the influence of innate immunity activators we used an artificial analogue of U25 box C/D snoRNA, and IFNa.

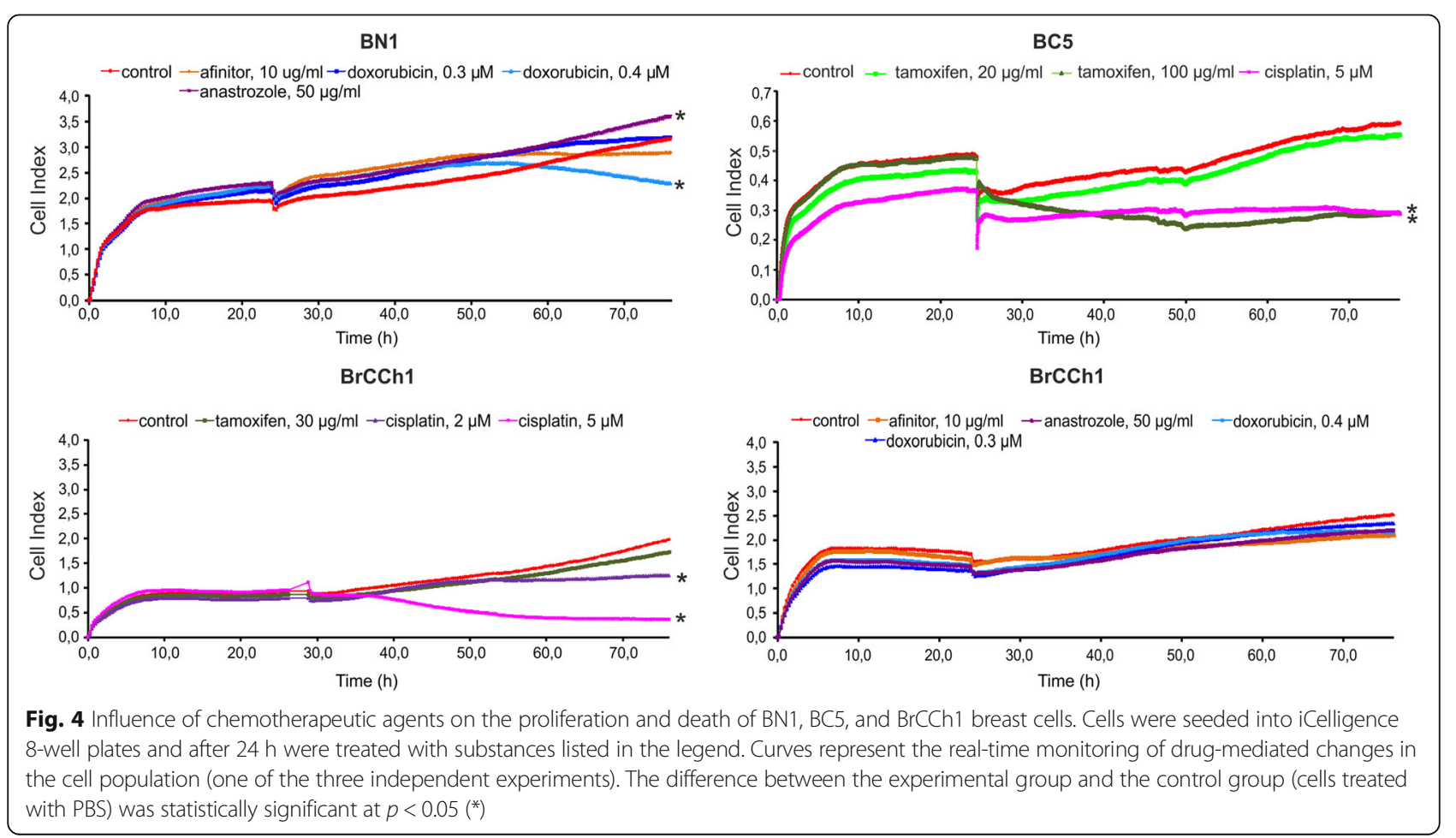


First, we analysed differences in sensitivity of primary and immortalized cells to the cytotoxic action of U25 snoRNA analogue and IFN $\alpha$. We estimated effective concentrations of the substances that provide the optimal level of changes in cells viability using iCelligence RTCA assay. It was determined that U25 snoRNA analogue and IFN $\alpha$ induced decrease in cell viability in the range of $\sim 5$ to $70 \%$ at concentrations $10 \mathrm{nM}$ and $220 \mathrm{UI} / \mathrm{mL}$ for U25 snoRNA analogue and IFNa, respectively (Fig. 5). The MCF-7 cells were the most responsive to the cytotoxic action of IFN $\alpha$ in the row MCF-7 > MCF10A > MDA-MB-231 (with viability of 63, 75 and 84\%, correspondently Fig. 5a). BC5, BrCCh1, and BN1 breast cells did not show statistically significant differences in the sensitivity to snoRNA analogue and IFN $\alpha$ (Fig. 5b).

Taking into account that the interferon- $\alpha$ has the pleiotropic biologic activities, i.e. does not necessarily imply the proliferative or apoptotic cellular response, we analysed the activation of innate immunity processes in BC5, BrCCh1, and BN1 cells and in the immortalized breast cells MCF-7, MDA-MB-231, and MCF10A. For this we incubated cells with an artificial analogue of U25 box C/D snoRNA or IFN $\alpha$ and estimated the level of IFIT3 mRNA using qRT-PCR.

We determined that MCF7 cells were the most sensitive to artificial snoRNA, evidenced by the 100 -fold increase in IFIT3 mRNA (Fig. 6a). IFIT3 mRNA was increased 30- and 20-fold following treatment with the immune-stimulating RNA (isRNA) in MDA-MB-231 cancer cells and MCF10A normal cells, respectively (Fig. 6a). It should be noted that the degree of changes in the IFIT3 mRNA level caused by artificial snoRNA decreased in the following order: MCF-7 > MDA-MB-231 > MCF10A. Treatment with IFN $\alpha$ revealed a similar sensitivity: 11-, 9-, and 2-fold changes in IFIT3 mRNA in MCF-7, MDA-MB-231, and MCF-10a cells, respectively. The increase in IFIT3 mRNA induced by IFN $\alpha$ was lower than that for snoRNA (Fig. 6a and b).

To explain the differences in the activation of IFN $\alpha$-inducible genes, we estimated the baseline level of IFIT3 mRNA and determined that the activation of expression was inversely associated with the baseline level of IFIT3 mRNA (Fig. 7). This suggests that the relative baseline level of IFIT3 mRNA allows prediction of the sensitivity of cancer cells to the influence of innate immune modulators.

To confirm our hypothesis, we compared the mRNA levels of IFIT3 in non-treated BC5, BrCCh1, and BN1 cells, as well as in the primary breast cells following exposure to artificial U25 box C/D snoRNA or IFN $\alpha$. The highest transcriptional activation of IFIT3 was found in BrCCh1 cells (82-fold for the artificial snoRNA and 7-fold for IFN $\alpha$; Fig. $6 \mathrm{~b}$ and Fig. 7b), while these cells are characterised with the lowest baseline level of IFIT3 mRNA. BC5 cancer cells and normal BN1 cells demonstrated lower transcriptional activation that was inversely associated with the baseline expression level of the gene namely the snoRNA analogue induced a 10- and 32-fold increase in IFIT3 mRNA, respectively. IFN $\alpha$ exposure led to 3.5-fold increase in IFIT3 mRNA in BN1 normal cells and no significant change in BC5 cancer cells (Fig. 6 and Fig. 7). The Spearman's rank correlation coefficient between the baseline level and the relative activation of IFIT3 mRNA expression for all cell lines used was estimated at $r=-0.94$ for the U25 snoRNA analogue as for IFN $\alpha(p<0.05)$. Overall, for the immortalized and primary breast cells tested, we confirmed that the lower the IFIT3 mRNA baseline level, the higher the degree of transcriptional activation under the influence of innate immunity activators.

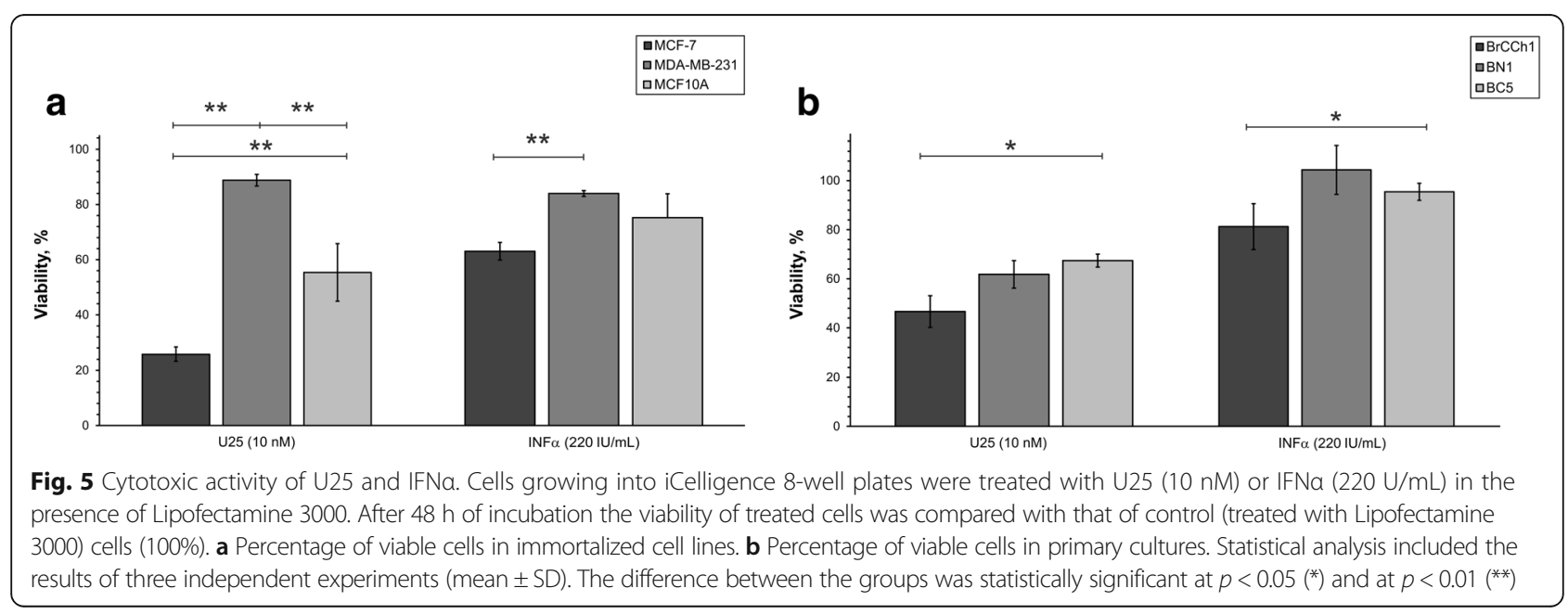



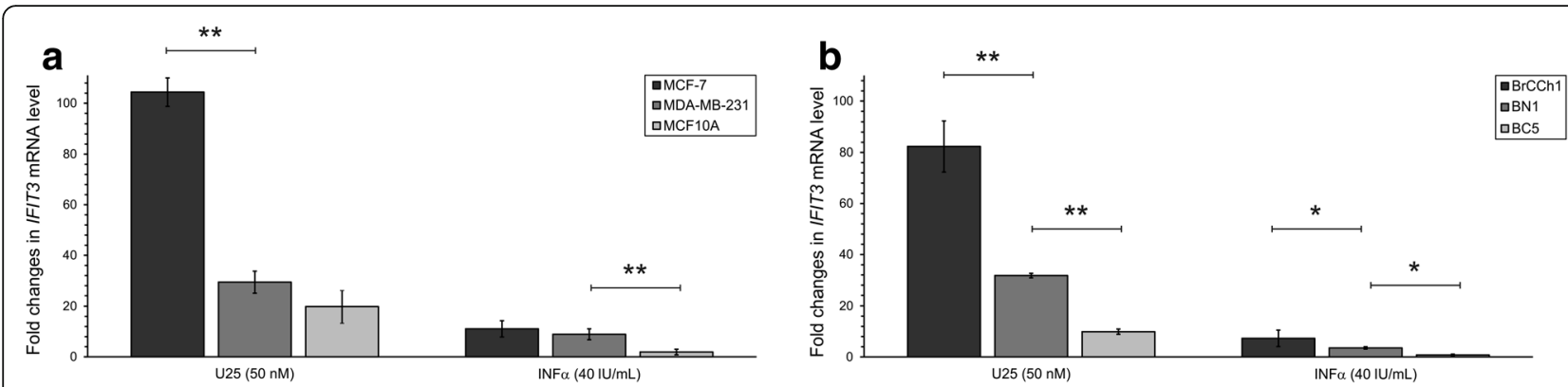

Fig. 6 Expression of IFIT3 gene in human breast cell lines: a MCF7, MDA-MB-231, MCF10A, and primary cell lines b BrCCh1, BN1, BC5 after $24 \mathrm{~h}$ transfection with an analog of U25 C/D box in the presence Lipofectamine 3000. Quantitative RT-PCR values were normalized to level of GAPDH, HPRT and RNU6 RNAs. Results are plotted relative to cells incubated with Lipofectamine 3000 for sample transfected with RNA and to non-treated cells for samples under INFa exposure. Data shown represent the mean \pm SD from two independent experiments. The difference between the groups was statistically significant at $p<0.05\left(^{*}\right)$ and at $p<0.01\left(^{* *}\right)$

\section{Discussion}

The present study focused on the establishment and characterisation of primary breast cancer and nonmalignant cell lines and their application as a model for comparative analyses of sensitivity to therapeutic treatments. It is known that for breast cancers the initial investigation of molecular markers, which are important for the choice of therapy, includes analysis of the expression of steroid hormone receptors as well as determining HER2 status [26].

Adjuvant hormone therapy is effective following surgery among patients with ER-positive and/or PGR-positive breast cancers. Meanwhile, women with $\mathrm{ER}^{-} / \mathrm{PGR}^{+}$or ER-poor/PGR ${ }^{-}$tumours also receive adjuvant anti-oestrogen hormone therapy with hope that this treatment will benefit this group of patients [27, 28]. In the case of IHC assays of ER and PGR cut point for "positive" is $1 \%$ of stained cells, $1-10 \%$ - weakly positive and $\geq 10 \%$ - high positive [29]. RT-qPCR also can be used for the determination of breast cancer molecular subtype by quantification of ER and PGR mRNA levels [30].

In the present study, we compared the sensitivity of established hormone-positive $\mathrm{BC} 5$ cells and poor hormone-positive BrCCh1 cells to tamoxifen. As expected, according to ER/PGR status, only hormone-positive BC5 cells were sensitive to tamoxifen treatment (Fig. 4). Thus, we can conclude that PCR analysis of ER/PGR status in established cancer cell $\mathrm{s}$ predicted sensitivity to hormone therapy.

However, the clinical impact of CD24 and CD44 expression in tumours remains unclear, and further investigation will be necessary to evaluate the correlation between ER expression and $\mathrm{CD} 44^{+} / \mathrm{CD} 24^{-}$cells in breast cancer. In general, $\mathrm{CD} 44^{+} / \mathrm{CD} 24^{-}$cells appear most commonly in the TNBC subtype. Basal marker expression can complement with $\mathrm{CD} 44^{+} / \mathrm{CD} 24^{-}$CSCs for an improved indicator for poor prognosis [28]. On the contrary, Horimoto and co-authors demonstrated that ER-positive patients with $\mathrm{CD} 44^{+} / \mathrm{CD} 24^{-}$tumours had
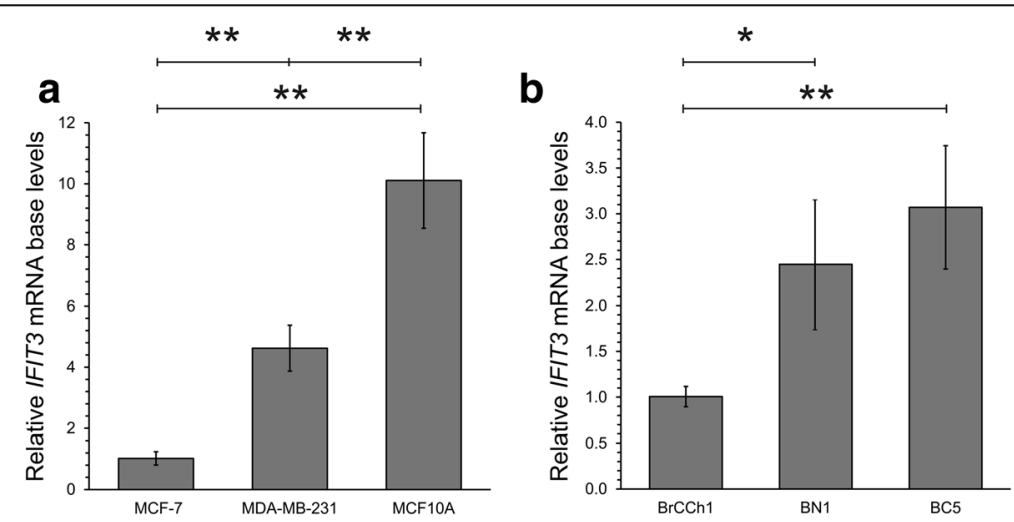

Fig. 7 Baseline IFIT3 mRNA level in human breast cell lines: a MCF7, MDA-MB-231, MCF10A, and primary cell lines b BrCCh1, BN1, BC5. Quantitative RT-PCR values were normalized to level of GAPDH, HPRT and RNU6 RNAs. a Results for MDA-MB-231 and MCF10A are presented as relative to level in MCF-7 cells; $\mathbf{b}$ results for BN1 and BC5 are presented as relative to level in BrCCh1 cells. Data shown represent the mean \pm SD from two independent experiments. The difference between the groups was statistically significant at $p<0.05\left(^{*}\right)$ and at $p<0.01\left(^{* *}\right)$ 
significantly longer disease-free survival than all other ER-positive patients [31]. Here, we observed high contribution of the $\mathrm{CD} 44^{+} / \mathrm{CD} 24^{-}$population in the BrCCh1 ER-poor/PGR-poor/Her2 ${ }^{\text {low }}$ tumour cell line, in which the phenotype is relatively close to TNBC (Fig. 3). Given the putative role of CSCs in tumour development and recurrence, we suppose that the low doxorubicin sensitivity of $\mathrm{BrCCh} 1$ cells that originated from the recurrent tumour is caused by CSC-like $\mathrm{CD} 44^{+} / \mathrm{CD} 24^{-}$cells. BrCCh1 cells were also insensitive to tamoxifen, anastrozole, and afinitor, thus, it will be important to screen additional therapeutics to improve anticancer therapies for aggressive types of cancer. Epithelial-to-mesenchymal transition (EMT) has been described taking place in epithelial cells of breast cancers in vivo and in vitro [32]. EMT is visually defined by emergence of a fibroblastic-like cell morphology. Heterogeneous morphology of established $\mathrm{BrCCh} 1$ and $\mathrm{BC} 5$ cells, which were presented by flattened mesenchymal-like cells and epithelioid cells, can support thesis that during cultivation some of these cells undergo epithelial-to-mesenchymal transition. Such transition can be induced by CSCs, which have been detected in BrCCh1 and BC5 cells.

Activators of the immune system could become new powerful amplifiers of traditional chemotherapeutics for destroying cancers with poor prognosis [33]. On the other hand, artificial analogues of snoRNAs were recently shown to activate innate immunity in cancer cells $[14,34]$. The action of isRNAs and interferons on cells can be described as results of interaction between ligands (RNAs or interferon) and receptors (Fig. 8). It is known, that interferon- $\alpha$ binds to the interferon- $\alpha / \beta$

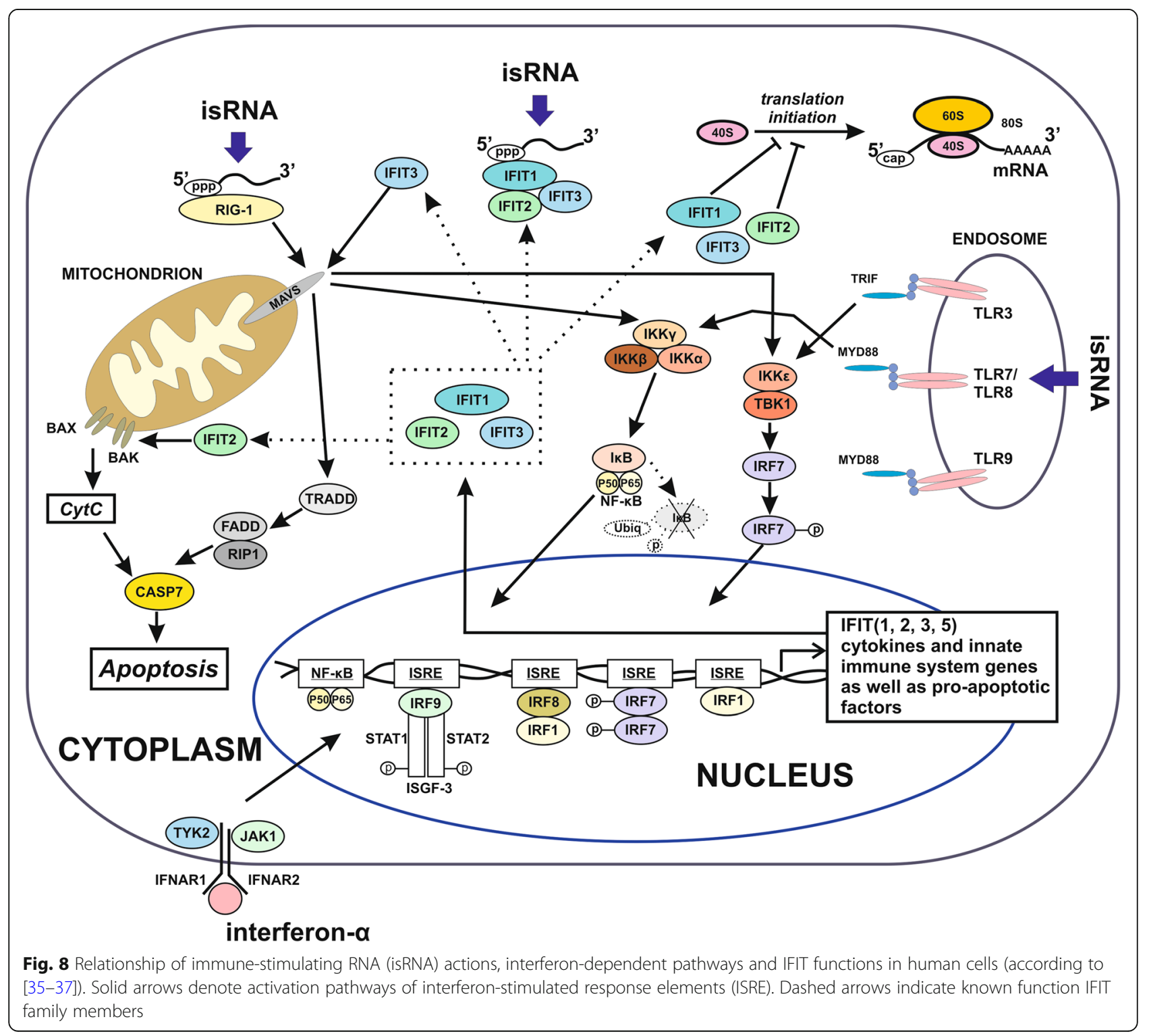


receptor (IFNAR) and activates interferon-dependent cascades. Activation of the expression of interferon-sensitive genes in mammalian cells by RNA analogues is provided by a set of RNA-binding receptors, including TLRs, RIG-I, MDA-5, and PKR [35]. Earlier we found that artificial box $\mathrm{C} / \mathrm{D}$ snoRNAs induced strong innate immune response. In particular, they upregulated transcription of the genes involved in cellular response to viral infection and foreign genetic material, such as RIG-I (DDX58), OAS1, MYD88, RNASEL, PKR (EIF2AK2), as well as interferon-dependent transcription factor STAT and IRF families (Fig. 8). Some products of interferon-activated genes are involved in cell death pathways and such activation can result in decrease of cell viability [14, 35-37].

In the present study, we analysed the effects of an artificial analogue of U25 snoRNA as well as INF $\alpha$ on IFIT3 gene expression in primary breast cells and immortalized breast cell lines. We revealed that the activation of IFIT3 expression was inversely correlated with its mRNA baseline level (Fig. 6 and Fig. 7).

As described by Zhao and co-authors, elevated expression of IFIT3 enhanced anti- apoptotic activity and chemotherapy resistance in pancreatic ductal adenocarcinoma [38]. Our data demonstrate that a BrCCh1 chemoresistant cells with a large contribution of CSC-like population exhibits low baseline IFIT3 mRNA with a prominent response to stimulation with snoRNA analogue or IFN $\alpha$. On the contrary, nonmalignant breast MCF10A cells expressed relatively high IFIT3 mRNA in the culture conditions, compared with MCF7 and MDA-MB-231 cells, and demonstrated modest response to xenogeneic RNA and INF $\alpha$.

Yang studied the expression of IFIT3 in hepatocellular carcinoma specimens following IFN therapy and showed that the activation of IFIT3 expression correlates with patient survival [15]. In our study, comparing the data of IFIT3 expression within particular cell lines following stimulation by immunomodulators and without stimulation we concluded that there was an inverse correlation in the intensity of the IFIT3 response to immunostimulators and constitutive IFIT3 mRNA expression. Elevated level of IFIT3 in cancer cells can be regarded as alteration in activation pathways as well as known IFIT3-dependent downstream signalling (Fig. 8). It should be noted that the IFIT3 level can be used as an indicator of cancer cells with null or low sensitivity to immune-stimulating agents. To establish molecular mechanisms determining these phenotypes of cancer cells the studies of mutations of IFIT3 and its partner genes, copy number variations and transcription factor activity are needed.

\section{Conclusions}

Here, we studied three primary cultures after propagation from human breast tissue and compared their response to conventional chemotherapeutics and to innate immunity stimulators with that of the immortalized breast cells MCF7, MDA-MB-231, and MCF10A. Our results suggest that when treated with xenogeneic RNA and INF $\alpha$, the intensity of IFIT3 mRNA expression is inversely associated with the baseline level of this mRNA in breast cancer cells. Summarizing our results and recently reported data, IFIT3 expression can be considered a prognostic indicator of the efficacy of cancer immunotherapy. Because we did not observe any correlation of IFIT3 expression and the ER/PGR/HER2 status of cancer cells, we suggest that IFIT3 expression be tested regardless of ER/PGR/HER2 status if innate immunity activators will be used.

\section{Abbreviations \\ ASCO: American Society of Clinical Oncology; CSC: Cancer stem cells; ER: Estrogen receptors; HER: Human epidermal growth factor receptors; IFIT3: Interferon-induced protein with tetracopeptide repeat 3; INF: Interferons; isRNA: Immune-stimulating RNA; PGR: Progesterone receptors; TNBC: Triple negative breast cancer}

\section{Funding \\ This work was funded by Russian Science Foundation Grant RSF 16-14- 10284 and partially supported by RFBR grant 16-34-60136.}

\section{Availability of data and materials}

The data that support the findings of this study are available from the authors upon reasonable request.

\section{Authors' contributions}

OK, AN, VR and GS designed the study. Primary cultures were developed by AN. AN, EJ, EB, AG, SS, ES, and EK performed the experiments. OK, AN, DS and GS analyzed the data. The draft manuscript was prepared by OK with input from DS, AN, GS, EK and VR. All authors agreed the final version. All authors read and approved the final manuscript.

Ethics approval and consent to participate

All patients gave written informed consent. The study protocol was approved by the Institute of Molecular Biology and Biophysics SB RAMS Ethics Committee in accordance with the Declaration of Helsinki of 1975.

\section{Consent for publication}

Not applicable.

\section{Competing interests}

The authors declare that they have no competing interests.

\section{Publisher's Note}

Springer Nature remains neutral with regard to jurisdictional claims in published maps and institutional affiliations.

\section{Author details}

${ }^{1}$ Institute of Chemical Biology and Fundamental Medicine, Siberian Branch, Russian Academy of Sciences, Lavrentiev Avenue, 8, 630090 Novosibirsk, Russia. ${ }^{2}$ Novosibirsk State University, Pirogova str., 1, 630090 Novosibirsk, Russia. ${ }^{3}$ National Novosibirsk Regional Oncology Dispensary, Plakhotnogo str., 2, 630000 Novosibirsk, Russia. ${ }^{4}$ Novosibirsk Municipal Budgetary Healthcare Institution "Municipal Clinical Hospital \#1", Zalessky str., 6, 630047 Novosibirsk, Russia. ${ }^{5}$ Center of New Medical Technologies, Pirogova, str., 25/4, 630090 Novosibirsk, Russia. 
Received: 10 November 2017 Accepted: 25 June 2018 Published online: 09 July 2018

\section{References}

1. Siegel RL, Miller KD, Jemal A. Cancer statistics. CA Cancer J Clin. 2016;66:7-30.

2. Schilsky RL. Personalized medicine in oncology: the future is now. Nat Rev Drug Discov. 2010:9:363-6.

3. Rivenbark AG, O'Connor SM, Coleman WB. Molecular and cellular heterogeneity in breast cancer: challenges for personalized medicine. Am J Pathol. 2013;183:1113-24.

4. Singhal $H$, Greene $M E$, Tarulli $G$, Zarnke $A L$, Bourgo RJ, Laine $M$, et al, Genomic agonism and phenotypic antagonism between estrogen and progesterone receptors in breast cancer. Sci Adv. 2016;2:e1501924.

5. Liu S, Chia SK, Mehl E, Leung S, Rajput A, Cheang MCU, et al. Progesterone receptor is a significant factor associated with clinical outcomes and effect of adjuvant tamoxifen therapy in breast cancer patients. Breast Cancer Res Treat. 2010;119:53-61.

6. Von Minckwitz G, Untch M, Blohmer JU, Costa SD, Eidtmann H, Fasching PA, et al. Definition and impact of pathologic complete response on prognosis after neoadjuvant chemotherapy in various intrinsic breast cancer subtypes. J Clin Oncol. 2012;30:1796-804.

7. Hussenet T, Dembélé D, Martinet N, Vignaud JM, Du Manoir S. An adult tissuespecific stem cell molecular phenotype is activated in epithelial cancer stem cells and correlated to patient outcome. Cell Cycle. 2010;9:321-7.

8. Oon ML, Thike AA, Tan SY, Tan PH. Cancer stem cell and epithelialmesenchymal transition markers predict worse outcome in metaplastic carcinoma of the breast. Breast Cancer Res Treat. 2015;150:31-41.

9. Geeleher P, Cox NJ, Huang R. Clinical drug response can be predicted using baseline gene expression levels and in vitro drug sensitivity in cell lines. Genome Biol. 2014;15:R47.

10. Llovet JM, Sala M, Castells L, Suarez Y, Vilana R, Bianchi L, et al. Randomized controlled trial of interferon treatment for advanced hepatocellular carcinoma. Hepatology. 2000;31:54-8.

11. Uenishi T, Kubo S, Hirohashi K, Tanaka H, Shuto T, Yamamoto T, et al. Relationship between response to previous interferon therapy and postoperative recurrence of hepatitis C virus-related hepatocellular carcinoma. Hepatol Res. 2002;24:404-12.

12. Warren KE, Young HA. Interferon therapy for malignant solid tumors. Curr Drug Ther. 2010;5:132-8.

13. Van DN, Roberts CF, Marion JD, Lepine S, Harikumar KB, Schreiter J, et al. Innate immune agonist, dsRNA, induces apoptosis in ovarian cancer cells and enhances the potency of cytotoxic chemotherapeutics. FASEB J. 2012 26:3188-98.

14. Stepanov GA, Filippova JA, Nushtaeva AA, Kuligina EV, Koval OA, Richter VA, et al. Artificial analogues of circulating box C/D RNAs induce strong innate immune response and microRNA activation in human adenocarcinoma cells. Adv Exp Med Biol. 2016:924:121-5.

15. Yang Y, Zhou Y, Hou J, Bai C, Li Z, Fan J, et al. Hepatic IFIT3 predicts interferon-a therapeutic response in patients of hepatocellular carcinoma. Hepatology. 2017:66:152-66.

16. Koval OA, Sakaeva GR, Fomin AS, Nushtaeva AA, Semenov DV, Kuligina EV, et al. Sensitivity of endometrial cancer cells from primary human tumor samples to new potential anticancer peptide lactaptin. J Cancer Res Ther. 2015:11:345-51.

17. Livak KJ, Schmittgen TD. Analysis of relative gene expression data using real-time quantitative PCR and the 2(-Delta Delta C (T)) method. Methods. 2001:25:402-8

18. Pfaffl MW. A new mathematical model for relative quantification in real-time RT-PCR. Nucleic Acids Res. 2001;29:45e-45.

19. Hellemans J, Mortier G, De Paepe A, Speleman F, Vandesompele J. qBase relative quantification framework and software for management and automated analysis of real-time quantitative PCR data. Genome Biol. 2007;8: R19.

20. Aceto N, Duss S, MacDonald G, Meyer DS, Roloff T-C, Hynes NE, et al. Coexpression of HER2 and HER3 receptor tyrosine kinases enhances invasion of breast cells via stimulation of interleukin-8 autocrine secretion. Breast Cancer Res. 2012;14:R131.

21. Wolff AC, Hammond ME, Hicks DG, Dowsett M, McShane LM, Allison KH, et al. Recommendations for human epidermal growth factor receptor 2 testing in breast cancer: American Society of Clinical Oncology/College of
American Pathologists clinical practice guideline update. TL - 31. J Clin Oncol. 2013:31:3997-4013.

22. Idowu MO, Kmieciak M, Dumur C, Burton RS, Grimes MM, Powers CN, et al. CD44(+)/CD24(-/low) cancer stem/progenitor cells are more abundant in triple-negative invasive breast carcinoma phenotype and are associated with poor outcome. Hum Pathol. 2012;43:364-73.

23. Dhillon S. Everolimus in combination with exemestane: a review of its use in the treatment of patients with postmenopausal hormone receptorpositive, her2-negative advanced breast cancer. Drugs. 2013;73:475-85.

24. Brueggemeier RW, Hackett JC, Diaz-Cruz ES. Aromatase inhibitors in the treatment of breast cancer. Endocr Rev. 2005:26:331-45.

25. Legrier ME, Bièche I, Gaston J, Beurdeley A, Yvonnet V, Déas O, et al. Activation of IFN/STAT1 signalling predicts response to chemotherapy in oestrogen receptor-negative breast cancer. Br J Cancer. 2016;114:177-87.

26. Banin Hirata BK, Oda JMM, Losi Guembarovski R, Ariza CB, De OCEC, MAE W. Molecular markers for breast cancer: prediction on tumor behavior. Dis Markers. 2014;2014:513158. https://doi.org/10.1155/2014/513158.

27. Fan $Y$, Ding $X, X u B, M a F$, Yuan $P$, Wang J, et al. Prognostic significance of single progesterone receptor positivity a comparison study of estrogen receptor negative/progesterone receptor positive/her2 negative primary breast cancer with triple negative breast cancer. Medicine (United States). 2015:94:e2066.

28. Tsang JY, Huang YH, Luo MH, Ni YB, Chan SK, Lui PC, et al. Cancer stem cell markers are associated with adverse biomarker profiles and molecular subtypes of breast cancer. Breast Cancer Res Treat. 2012;136:407-17.

29. Hammond MEH, Hayes DF, Dowsett M, Allred DC, Hagerty KL, Badve S, et al. American society of clinical oncology/college of american pathologists guideline recommendations for immunohistochemical testing of estrogen and progesterone receptors in breast cancer. J Clin Oncol. 2010;28:2784-95.

30. Laible M, Schlombs K, Kaiser K, Veltrup E, Herlein S, Lakis S, et al. Technical validation of an RT-qPCR in vitro diagnostic test system for the determination of breast cancer molecular subtypes by quantification of ERBB2, ESR1, PGR and MKI67 mRNA levels from formalin-fixed paraffin-embedded breast tumor specimens. BMC Cancer. 2016;16:398.

31. Horimoto Y, Arakawa A, Sasahara N, Tanabe M, Sai S, Himuro T, et al. Combination of cancer stem cell markers CD44 and CD24 is superior to ALDH1 as a prognostic indicator in breast cancer patients with distant metastases. PLoS One. 2016;11:e0165253.

32. Foroni C, Broggini M, Generali D, Damia G. Epithelial-mesenchymal transition and breast cancer: role molecular mechanisms and clinical impact. Cancer Treat Rev. 2012;38:689-97.

33. Jiang $X$, Shapiro DJ. The immune system and inflammation in breast cancer. Mol Cell Endocrinol. 2014;382:673-82.

34. Youssef OA, Safran SA, Nakamura T, Nix DA, Hotamisligil GS, Bass BL. Potential role for snoRNAs in PKR activation during metabolic stress. Proc Natl Acad Sci. 2015;112:5023-8.

35. Diamond MS, Farzan M. The broad-spectrum antiviral functions of IFIT and IFITM proteins. Nat Rev Immunol. 2013:13:46-57.

36. Ivashkiv LB, Donlin LT. Regulation of type I interferon responses. Nat Rev Immunol. 2014;14:36-49.

37. Fleith RC, Mears HV, Leong XY, Sanford TJ, Emmott E, Graham SC, et al. IFIT3 and IFIT2/3 promote IFIT1-mediated translation inhibition by enhancing binding to non-self RNA. Nucleic Acids Res. 2018; https://doi.org/10.1093/ nar/gky191.

38. Zhao Y, Altendorf-Hofmann A, Pozios I, Camaj P, Däberitz T, Wang X, et al. Elevated interferon-induced protein with tetratricopeptide repeats 3 (IFIT3) is a poor prognostic marker in pancreatic ductal adenocarcinoma. J Cancer Res Clin Oncol. 2017;143:1061-8. 